\title{
Las garrapatas silvestres como potenciales vectores de Leishmania sp.
}

\author{
Jesús Rojas-Jaimes ${ }^{I}$
}

\section{Resumen}

La leishmaniasis es una enfermedad endémica del Perú y descuidada desde el punto de vista de Salud Pública. El objetivo del presente trabajo fue evaluar nuevos artrópodos que podrían transmitir el parásito de la Leishmania. Se colectaron garrapatas de animales silvestres como Pecari tajacu y Chelonoidis denticulata del distrito de Las Piedras en Madre de Dios y se utilizó una prueba molecular para detectar el parasito de la Leishmania. Se identificó a las garrapatas de Pecari tajacu como Rhipicephalus microplus y a las colectadas de Chelonoidis denticulata como Amblyoma sabanerae. La detección molecular de Leishmania sp. fue un $95,7 \%$ para $R$. microplus y $88,9 \%$ para $A$. sabanerae. Los resultados nos indican una alta tasa de parasitemia en las garrapatas estudiadas, por tanto, estos datos son novedosos y promisorios como base en la búsqueda de nuevos probables vectores como las garrapatas y reservorios como podrían ser Pecari tajacu y Chelonoidis denticulata.

Palabras clave: Leishmaniasis, vectores, animales silvestres, garrapatas, Leishmania.

\section{Abstract}

Leishmaniasis is an endemic disease of Peru and neglected from the point of view of Public Health. The objective of the present work was to evaluate new arthropods that could transmit the parasite of Leishmania. Ticks from wild animals such as Pecari tajacu and Chelonoidis denticulata from the Las Piedras district of Madre de Dios were collected and a molecular test was used to detect the Leishmania parasite. Rhipicephalus microplus ticks was identified in Pecari tajacu and Amblyoma sabanerae in Chelonoidis denticulata. The molecular detection of Leishmania sp. it was $95.7 \%$ for R. microplus and $88.9 \%$ for A. sabanerae. The results indicate a high rate of parasitemia in the ticks studied, therefore these data are novel and promising as a basis in the search for new probable vectors such as ticks and reservoirs such as Pecari tajacu and Chelonoidis denticulata.

Keywords: Leishmaniasis, vectors, wild animals, ticks, Leishmania.

\section{Introducción}

La leishmaniasis es una enfermedad de importancia pública en la que el ciclo de transmisión del patógeno es complejo. Las medidas de control de la enfermedad son complicadas debido a la participación de varios factores en la enfermedad entre ellos las diferentes especies del vector, los diferentes reservorios y/o hospederos entre los cuales el ser humano y finalmente las condiciones climáticas y sociales ${ }^{(1)}$.

Se reporta que existen al menos 30 especies diferentes de Leishmania y solo un grupo de ellas pertenecientes al subgénero Viannia y Leishmania son patógenas para el ser humano $^{(2)}$.

En la actualidad existen diferentes marcadores utilizados para detectar e identificar las especies del género Leishmania; estos marcadores están localizados a nivel nuclear o mitocondrial y dentro de este pueden estar a nivel del maxicirculo o minicirculo ${ }^{(2)}$. Cada marcador dispone de ciertas ventajas referentes al otro como estabilidad, número de copias o polimorfismo ${ }^{(2)}$. Entre el marcador más usado está el ADN del kinetoplasto del parásito, aunque el diseño de los primeros y la verificación de secuencias homólogas y/o complementarias usando la bioinformática así como el tamaño del producto amplificado es necesario para evitar falsos $\operatorname{positivos}^{(3)}$.

En los últimos años el rol de los ectoparásitos como garrapatas, pulgas y piojos está mostrando interés por estar presentes en las áreas de leishhmaniasis visceral y por su rol de efectivos transmisores de patógenos, adicionalmente, varios estudios reportaron la presencia de los ácidos nucleicos de Leishmania en garrapatas por lo que se presume la competencia de estos artrópodos en el ciclo de la transmisión del agente causal de la leishmaniasis ${ }^{(1,4)}$.

Las garrapatas pertenecen a dos grandes familias: Ixodidae (garrapatas duras) y Argasidae (garrapatas blandas).

${ }^{\prime}$ Escuela de Medicina Humana, Área de Biología Celular y Molecular, Universidad Científica del Sur, Lima-Perú. Facultad de Ciencias, Universidad Continental, Lima-Perú. 
El género Rhipicephalus se encuentra dentro de la familia Ixodidae y estas pueden transmitir una serie de enfermedades como lyme, rickettsiosis y encefalitis ${ }^{(5)}$.

En $R$. sanguineus han sido reportados por biología molecular patógenos de importancia veterinaria y zoonotica como Ehrlichia canis, Hepatozoon canis y Leishmania infantum chagasi ${ }^{(6)}$. Un estudio en Brasil demostró que un $10,1 \%$ de $R$. sanguineus colectados de perros sintomáticos como asintomáticos fueron positivos para Leishmania infantum en el $12.2 \%$ de canes colectados en una zona endémica de leishmaniasis canina ${ }^{(7)}$.

El objetivo de nuestro estudio es la detección molecular de Leishmania $s p$ en garrapatas silvestres colectados de animales silvestres.

\section{Metodología}

\section{Aspectos éticos y trabajo de campo}

Esta investigación fue aprobada por la Oficina de Salud Pública y Medio Ambiente del Consejo Regional de Madre de Dios, Perú. En el estudio, las pruebas para la identificación de Leishmania sp. se completaron con procedimientos de laboratorio ajustados a las guías internacionales sobre la investigación con animales y las normas del Comité de Cuidado y Uso de Animales del Área de Investigación en Salud de la Junta del Consejo Regional de Madre de Dios.

\section{Colecta}

Se colectaron 26 garrapatas (13 machos, 9 hembras y 4 ninfas) clasificadas taxonómicamente como Rhipicephalus (Boophilus) microplus de tres Pecari tajacu "sajino" en del área de Botijón del distrito de Las Piedras en la provincia de Tambopata, Madre de Dios, Perú. Pueblo Botijón (1207'12,95"S, 6904'31,47"O; WGS 84, 267 msnm). Así mismo en el caso de la Localidad de Chacra Gamitana, distrito de Las Piedras, ubicada en la margen izquierda del río Bajo Madre de Dios (Este -502134, Norte 8617027- Coordenadas UTM) se realizaron las colectas manuales de Amblyomma sabanerae, tomadas de Chelonoidis denticulata "motelo" estas colectas se realizaron en el horario diurno de 9:00 a.m. a 11 a.m.

Las garrapatas fueron identificadas a través de claves taxonómicas usando el hipostoma, escudo, capítulo, coxas, tarso, placa espiracular y palpo.

\section{Biología molecular}

Se perdieron tres muestras en el proceso ( 2 hembras y 1 macho) de R. microplus y se utilizó un PCR basado en la identificación del ADN blanco para el gen de la subunidad pequeña del ARN ribosomal (SSU rRNA), el cual es conservado en todas las especies de Leishmania, y es de 160 copias en cada célula del parásito para detectar la presencia de ADN de Leishmania sp. la especificidad de la prueba fue dada por la sonda Taqman para Leishmania sp. ${ }^{(8) .}$ Los controles positivos usados fueron 5ASK ( $L$. major), Lc53 ( $L$. braziliensis) y como control negativo se usó el agua de grado biología molecular.

\section{Resultados}

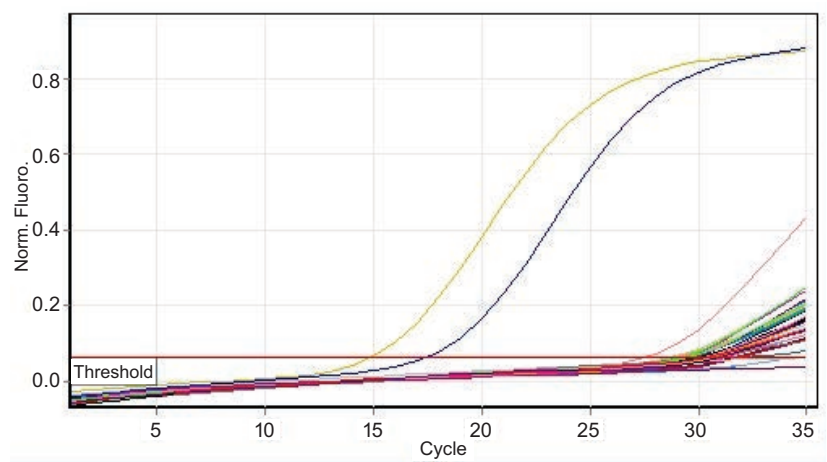

Figura 1. Curvas positivas del PCR en la detección de Leishmania.

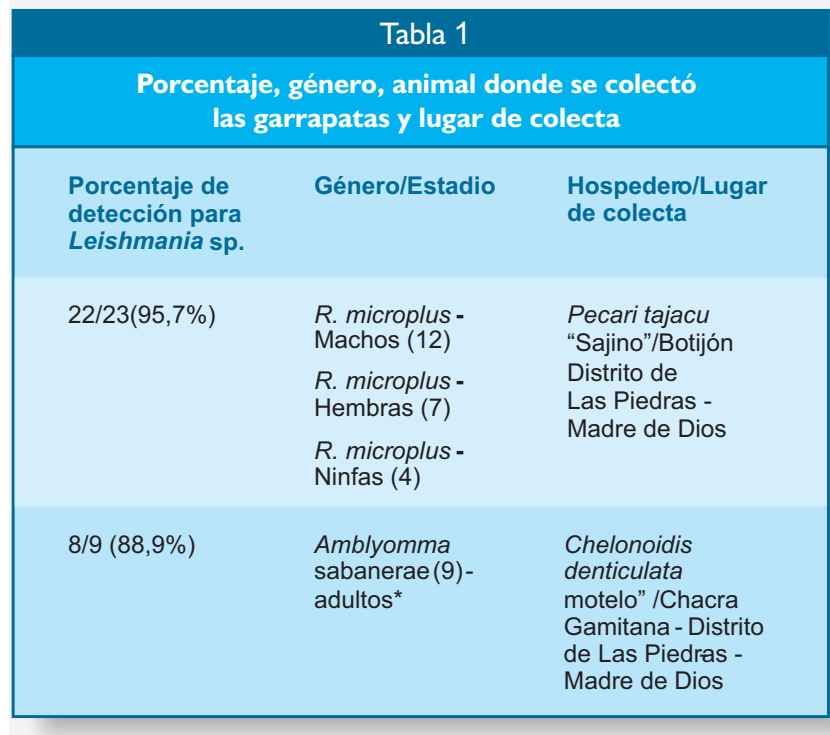

${ }^{*}$ No se identificó el género

\section{Discusiones}

Estudios previos han demostrado la presencia de $\mathrm{ADN}$ y ARN de L. infantum en garrapatas, pulgas y piojos ${ }^{(1)}$. La actividad humana intrusiva como la deforestación, la agricultura, la tala de la madera y la minería en los nichos del parasito es un factor de importancia en la epidemiología de la leishmaniasis tegumentaria ${ }^{(1)}$.

Los estudios moleculares para la identificación de especies basadas en amplificar los citocromos B, Cox II y C son útiles en combinación del secuenciamiento automatizado, 
aunque debemos remarcar que estos genes se encuentran a nivel del maxicirculo del kinetoplasto o a nivel de los cromosomas (genoma nuclear) con un número menor de copias cuando se contrasta al minicirculo del kinetoplasto el cual también ha sido usado en la identificación de especies ${ }^{(2)}$.

Estudios previos ya reportaron la ventaja del uso del minicirculo del kinetoplasto para identificar al género del parasito Leishmania, debido esto a su alto número de copias presente a nivel mitocondrial ${ }^{(2)}$. Adicionalmente otra investigación detectó la presencia del ADN del kinetoplasto de $L$. infantum en $R$. sanguineus a nivel de los intestinos y glándulas salivales además de aislar los parásitos en forma de promastigotes en medios de cultivo determinando la viabilidad de Leishmania ${ }^{(9)}$.

Es importante remarcar que, aunque no se ha demostrado aún el rol de las garrapatas como vectores donde se encuentra la forma de promastigote de la Leishmania excepto en un estudio, este artrópodo, así como otros dípteros diferentes de Lutzomyia sp. pudieran actuar como vectores mecánicos ${ }^{(4)}$.

En nuestro estudio $R$. microplus puede haberse parasitado al ingerir sangre contaminada de Pecari tajacu. así también pudo haberse dado con $A$. sabanerae al succionar la sangre de Chelonoidis denticulata. Ya un estudio previo ha demostrado la presencia de L. infantum chagasi a nivel de $R$. sanguineus así como en la sangre de perros por lo que se presume una relación de contaminación entre el artrópodo y hospedero o reservorio pueda ser del artrópodo al perro inoculando el parásito, del perro al artrópodo ingiriendo el parásito o en ambos sentidos ${ }^{(6)}$. En contraste nuestro estudio novedoso sin reportes previos sobre garrapatas colectadas de Pecari tajacu. y Chelonoidis denticulata positivas para Leishmania sp. lo que abre una nueva área base de estudio sobre probables nuevos vectores y reservorios de Leishmania sp.
En nuestro estudio en el caso de $R$. microplus colectados de Pecari tajacu la mayoría de garrapatas positivas para Leishmania fueron machos $(52,2 \%)$ y esto puede deberse a la necesidad del macho a buscar pareja y buscar varios hospederos en busca de alimento según se contrasta en un estudio anterior donde se encontró $92,3 \%$ de machos de $R$. sanguineus parasitados respecto al 7,7\% de hembras para Leishmania infantum respecto al total de garrapatas parasitadas (7). En el caso de $A$. sabanerae un $88,9 \%$ fue positivo molecularmente para Leishmania sp.

Es de suma importancia remarcar que el $95,7 \%$ de $R$. microplus y el $88,9 \%$ de $A$. sabanerae estudiados fueron positivos para Leishmania, resaltando el alto porcentaje de infestación parasitaria en ambos géneros de garrapatas. Lo que hace suponer la probabilidad vectorial de estas y que Pecari tajacu y $A$. sabanerae pudieran comportarse como reservorios, recordando que un estudio previo ha demostrado la presencia de ADN de Leishmania y L. infantum en las glándulas salivales de las garrapatas como $R$. sanguineus ${ }^{(7)}$. En contraste en un estudio anterior solo el $10,1 \%$ de $R$. sanguineus fueron positivos para $L$. infantum colectado de perros, aunque el estudio también remarca que a pesar del bajo porcentaje de garrapatas parasitadas existe una alta carga parasitaria en cada una de ellas ${ }^{(7)}$, siendo los resultados de nuestro estudio diferente, encontrándose un alto porcentaje de garrapatas parasitadas por artrópodo.

Se concluye que el porcentaje de $R$. microplus y $A$. sabanerae parasitado fue alto, además de inferir la posibilidad de que Pecari tajacu y Chelonoidis denticulata que fueron los animales de donde se colectaron las garrapatas pudieran comportarse como un probable reservorio. Por tanto se hace necesario otros estudios para dilucidar al detalle el vínculo del reservorio-artrópodo-hospedero en el ciclo de la leishmaniasis siendo nuestros hallazgos una base promisoria en este campo.

Referencias bibliográficas

1. Morais RCS De, Gonçalves-de-Albuquerque SDC, Silva RôPE, Costa PL, Silva KG Da, Silva FJ Da, et al. Detection and quantification of Leishmania braziliensis in ectoparasites from dogs. Vet Parasitol. 2013;196(3-4):506-508.

2. Lopes EG, Alberto C, Junior G, Marcili A, Silva RD. Performance of conventional pers based on primers directed to nuclear and mitochondrial genes for the detection and identification of Leishmania spp. 2016;(1).

3. Vergel C, Walker J, Saravia NG. Amplification of human DNA by primers targeted to Leishmania kinetoplast DNA and post-genome considerations in the detection of parasites by a polymerase chain reaction. Am J Trop Med Hyg. 2005;72(4):423-429.

4. Coelho WMD, Bresciani KDS. Molecular and parasitological detection of Leishmania spp. in a dipteran of the species Tabanus importunus. Rev Bras Parasitol Vet [Internet]. 2013;22(4):605-7. Available from: http://www.scielo.br/scielo.php?script=sci_arttext\&pid=S198429612013000400605\&lng=en\&nrm=iso\&tlng=en

5. Chen Z, Liu Q, Liu J-Q, Xu B-L, Lv S, Xia S, et al. Tick-borne pathogens and associated co-infections in ticks collected from domestic animals in central China. Parasit Vectors [Internet]. 2014;7(1):237. Available from: http://parasitesandvectors.biomedcentral.com/articles/10.1186/17563305-7-237

6. Gonçalves LR, Filgueira KD, Ahid SMM, Pereira JS, Vale AM Do,
Machado RZ, et al. Study on coinfecting vector-borne pathogens in dogs and ticks in Rio Grande do Norte, Brazil. Rev Bras Parasitol Veterinária [Internet]. $2014 ; 23$ (3):407-12. Available from: http://www.scielo.br/scielo.php?script $=$ sci arttext\&pid $=$ S 1984 29612014000300407\&lng=en\&tlng=en

7.Solano-Gallego L, Rossi L, Scroccaro A, Montarsi F, Caldin M, Furlanello T, et al. Detection of Leishmania infantum DNA mainly in Rhipicephalus sanguineus male ticks removed from dogs living in endemic areas of canine leishmaniosis. Parasit Vectors [Internet]. 2012;5(1):98. Available from: http://parasitesandvectors.biomedcentral.com/articles/ 10.1186/1756-3305-5-98

8.Van Eys, G.J., Schoone, G.J., Kroon, N.C., Ebeling, S.B., Sequence analysis of small subunit ribosomal RNA genes and its use for detection and identification of Leishmania parasites. Molecular and Biochemical Parasitology. 1992; 51:133-142.

9.Medeiros-Silva V, Gurgel-Gonçalves R, Nitz N, Morales LEDA, Cruz LM, Sobral IG, et al. Successful isolation of Leishmania infantum from Rhipicephalus sanguineus sensu lato (Acari: Ixodidae) collected from naturally infected dogs. BMC Vet Res [Internet]. BMC Veterinary Research; 2015;11(1):258. Available from: http://www.biomedcentral.com/1746 $6148 / 11 / 258$ 